\title{
Hepatoblastoma survival and the prognostic role of cancer stem cell markers
}

\author{
Mohamed Fawzy ${ }^{1 *}$, Abeer Bahnassy ${ }^{2}$, Mohamed El-Wakil $^{3}$, Ahmed Abdel-Sayed ${ }^{4}$ \\ ${ }^{I}$ Department of Pediatric Oncology, National Cancer Institute (NCI), Cairo University, Egypt. \\ ${ }^{2}$ Pathology Department, NCI, Cairo University, Egypt. \\ ${ }^{3}$ Department of Clinical Oncology, Faculty of Medicine Beni-Suef University, Egypt. \\ ${ }^{4}$ Department of Surgical Oncology, NCI, Cairo University, Egypt.
}

Received October 19, 2013; Revised December 04, 2013; Accepted December 05, 2013; Published Online December 15, 2013

\section{Original Article}

\begin{abstract}
Purpose: Hepatoblastoma (HB) is an embryonal tumor of the liver that occurs in infants and young children. Complete surgical resection and cisplatin-containing chemotherapy are crucial for cure in HB. Cancer stem cells (CSCs) constitute a newly identified subpopulation, which may differentiate into heterogeneous progenies of malignant cells. The aim of this study was to assess the survival outcome and the prognostic value of CSCs markers (CD133, CD90 and CD44) in a cohort of HB patients from Egypt. Methods: Disease status of $43 \mathrm{HB}$ patients was evaluated at the main checkpoints of therapy and during follow-up. Treatment included surgical tumor resection and systemic chemotherapy (cisplatin, 5-fluorouracil, doxorubicin, and vincristine). Protein and RNA expressions of CD44, CD 90 and CD 133 were assessed by immunohistochemistry and quantitative PCR. Results: The OS for all patients was 58.2 at 4 years. Patients with localized disease stages (I\&II) had a better OS than those with advanced stages (III\&IV) $(81.9 \%$ versus $30 \%, \mathrm{p}<0.001)$. Total surgical resection was superior to incomplete/no resection $(83.8 \%$ versus $25.2 \% ; \mathrm{p}<0.001)$. The OS was significantly correlated with tumor response ( $<<0.001)$ and each of CD44, CD 90, CD 133 expression $(\mathrm{p}<0.001)$ whereas reduced DFS was associated with CD44 and CD133 expression $(\mathrm{p}<0.001)$. Conclusion: Localized disease is associated with higher OS than more advanced stages III and IV. Complete surgical resection facilitated with systemic preoperative chemotherapy in initially irresectable cases can improve survival in HB while CSC markers (CD133, 44, and 90) can predict survival and response to treatment in HB patients.
\end{abstract}

Keywords: Hepatoblastoma; Survival; CSCs; Prognosis

\section{Introduction}

Benign and malignant liver tumors account for $1-2 \%$ of all pediatric tumors. However, recent data suggest that their incidence may be increasing at 5\% annually. ${ }^{1-3}$ Hepatoblastoma $(\mathrm{HB})$ is a rare embryonal tumor of the liver forming the most common childhood liver cancer in infants and young children with a median age of 16 months at diagnosis., ${ }^{4,5} \mathrm{~A}$ birth weight less than 1,000 grams is associated with strongly increased risk of $\mathrm{HB}$, while a moderately increased risk of

*Corresponding author: Mohamed Fawzy, MD; Department of Pediatric Oncology, National Cancer Institute, Cairo University and Children's Cancer Hospital-Egypt, Egypt.

Email: mfawzyonc@hotmail.com

Cite this article as:

Fawzy M, Bahnassy A, El-Wakil M, Abdel-Sayed A.

Hepatoblastoma survival and the prognostic role of cancer stem

cell markers. Int J Cancer Ther Oncol 2014; 2(1):02011.

DOI: $10.14319 /$ ijcto.0201.1 hepatoblastoma was found with younger maternal age, maternal smoking, infertility medications, and with higher maternal pregnancy body mass index. ${ }^{6}$ Ninety percent of patients with HB have a serum tumor marker alpha fetoprotein (AFP), which correlates with disease activity. Both AFP level at diagnosis and its rate of declining during treatment should be compared to the corresponding age-adjusted values. ${ }^{7}$

Both complete surgical resection and cisplatin-containing chemotherapy are crucial in achieving cure in HB. Radical resection can be obtained either by partial hepatectomy or with orthotopic liver transplant (OLT). ${ }^{8}$ Several histological subtypes of HB are known, including the wholly epithelial tumors, with pure fetal and mixed fetal/embryonal histology; mixed epithelial and mesenchmyal tumors; and several types of transitional, small and large cell undifferentiated tumors. ${ }^{9}$ This heterogeneity may explain their variable clinical behavior and reflects the distinct patterns of hepatic embryogenesis suggesting stem cell origin. ${ }^{10}$ 
Cancer stem cells (CSCs) are newly identified subpopulations isolated from several tumor types that possess stem cell properties and may differentiate into heterogeneous progenies of malignant cells. They are probably the progenitor cells that undergo unknown genetic mutations and lose potential for tissue repair, but retain stem cell characteristics, such as self-renewal and plasticity to differentiate into different cell types in tumor tissues. CSCs thought to be responsible for tumor development, resistance to treatment, metastasis and relapse. A better understanding of CSC pathobiology may aid in developing novel directed therapies against such cells. ${ }^{10-12}$

Few reports addressed the role of CSCs in HB. Some markers as CD133 and CD44 were used to detect HB-CSCs in cultured human HB cells, while CD90 was reported to be a CSC marker in hepatocellular carcinoma. ${ }^{13-14}$ CD133; a cell surface glycoprotein (prominin-1) that has been used as a marker for immature hematopoietic stem cells or progenitor cells while CD90 (Thy-1) is a glysyl-phosphatidyl-inosital (GPI)-anchored glycoprotein expressed in bone marrow-derived mesenchymal stem cells and hepatic stem cells progenitors. CD44 is a cell surface adhesion molecule mediating multiple signaling pathways which has been used as a CSC marker in leukemia, head and neck cancer, pancreatic, breast, and prostate cancer. ${ }^{15}$ The aim of this study was to evaluate the outcome in a cohort of Egyptian children with $\mathrm{HB}$ and to define the prognostic predictive value of CSCs markers (CD133, CD90 and CD44) in relation to survival, response to treatment and other prognostic factors.

\section{Patients and Methods}

\section{Patients}

This study included 43 HB patients who were diagnosed and treated at the Pediatric Oncology Department, National Cancer Institute (NCI), Cairo University between 2007 and 2012. All patients were newly diagnosed children below the age of 18 years on diagnosis, with HB of any stage, and were not previously treated elsewhere. Study protocol was approved by The Institutional Review Board (IRB) of the NCI which was in accordance with the 2007 declaration of Helsinki.

\section{Workup and Evaluation}

Histopathology and survival data of the patients under study were systematically reviewed and verified. Full history review, clinical examination and laboratory tests were done including complete blood picture, liver and kidney functions, and alpha-fetoprotein (AFP). Abdominal ultrasound and enhanced computerized tomography (CT) of the abdomen and pelvis were essentially done as baseline for all patients to determine the extent of involved parenchyma, and the presence or absence of macro vascular compression, displacement or invasion. The radiographic appearance of the tumor at diagnosis "PRETEXT" was used to assign the pretreatment extent of the tumor and "POST-TEXT" for patients received preoperative chemotherapy. ${ }^{16}$ All patients had also baseline CT chest and bone scan as part of their disease staging according to the Children's Oncology Group (COG) staging system as all stages from I to IV were represented among our patients. ${ }^{17}$ Evaluation of disease response was reviewed using Response Evaluation Criteria in Solid Tumors (RECIST). ${ }^{18}$ Tumor marker AFP level and imaging studies were carried out at the main checkpoints of therapy; post induction treatment, following definitive surgery, by end of treatment protocol and at regular intervals during follow up or as elsewhere required for evaluating a suspected event (progression/recurrence).

\section{Treatment}

Therapeutic approach included surgical resection of the primary tumor (segmentecomy/lobectomy) either at initial presentation whenever technically feasible or following upfront chemotherapy for inoperable tumors. Combined chemotherapy in form of cisplatin, 5-fluorouracil, doxorubicin and vincristine was given on adjuvant basis for patients with initially resected tumors (non-pure fetal stage I or positive margin stage II disease), or as neoadjuvant treatment for stage III and stage IV patients in accordance to COG guidelines. ${ }^{19}$ Lacking the facility and affordability, OLT was not an option in any of the studied patients.

\section{Immunohistochemistry (IHC)}

For each case, the most representative paraffin block was identified by examination of hematoxylin and eosin $(\mathrm{H} / \mathrm{E})$ stained slides. Determination of tumor: normal cell ratio was done for each sample and only samples including $\geq 75 \%$ neoplastic cells in the sections were selected. From each tumor block, $4 \mu \mathrm{m}$ thick sections ( 3 sections) were cut onto positive charged slides to be used for IHC analysis. Another $5 \mu \mathrm{m}$ thick sections (5 sections) were cut into a sterile Eppindorff tube for molecular studies. Slides were de-paraffinized in xylene followed by a series of graded ethanol. Antigen retrieval was performed by pressure cooking for 2 minutes in citrate buffer pH6.0. Endogenous peroxidases were blocked with $0.3 \%$ hydrogen peroxide and non-specific binding was blocked with normal goat serum. The cells were then reacted for $24 \mathrm{~h}$ at $40 \mathrm{C}$ with the primary antibody to: CD133 (mouse anti- human CD133, 1:500), CD90 (mouse anti-human CD90, 1:100) and CD44 (mouse anti- human CD44, 1:50) (all from Abcam, MA, USA); then with the secondary antibody (EnVision System/HRP, Dako, Tokyo, Japan) for 1 hour. After 
washing with PBS, sections were colored with $\mathrm{DAB}$, counterstained with hematoxylin and examined microscopically. Cases of breast and hepatocellular carcinomas were used as positive controls. A negative control was obtained by omitting the primary antibodies. The staining intensity was noted but not factored, as differing age of donor blocks and variation in fixation methods can impact on staining intensity. A case was considered positive, for CD133, CD44 or CD90, if brown membranous and/or cytoplasmic immunostaining was detected in $>10 \%$ of the cells. ${ }^{13-14}$

\section{RNA Extraction}

RNA was extracted from the representative sections of the tumor and normal tissues. Total RNA was isolated using RNeasy Mini Kit (Qiagen, Milan, Italy) and retro-transcribed using iScript TM cDNA Synthesis Kit (Bio-Rad, Milano, Italy) according to manufacturer's instructions. Extracted RNA was dissolved in diethyl pyrocarbonate-treated water containing $10 \mathrm{mmol} / \mathrm{l}$ of $\mathrm{MgCl} 2$ and incubated with $100 \mu \mathrm{g} / \mathrm{ml}$ of RNase-free DNase I for 30 minutes at $37^{\circ} \mathrm{C}$ to eliminate contaminated DNA. The reaction was stopped by heating the RNA at $95^{\circ} \mathrm{C}$ for 5 minutes after the addition of EDTA to a final concentration $30 \mathrm{mmol} / \mathrm{l}$.

\section{Quantitative real-time PCR (q-RT-PCR)}

Real-time quantitative (q-RT-PCR) analysis was performed with the SYBR Green PCR Master Mix using a stratagene MAX3000P according to the manufacturer's instructions (Applied Biosystems, Inc., Foster City, CA, USA). Primer sequences for CD133, CD44, and $\beta$-actin were as follows: CD133 (sense, GCTTTGCAATCTCCCTGTTG; antisense, TTGATCCGGGTTCTTACCTG), CD44 (sense, CGGACACCATGGACAAGTTT; antisense, CACGTGGAATACACCTGCAA), and $\beta$-actin (sense, ACAGAGCCTCGCCTTTGC; antisense, GCGGCGATA TCATCATCC).

PCR was performed in a final volume of $25 \mu \mathrm{l}$ with a SYBR Green PCR Master Mix using $1 \mu \mathrm{l}$ cDNA, $400 \mathrm{nM}$ of each primer for the respective genes. Cycling conditions were $50^{\circ} \mathrm{C}$ for 2 minutes and $95^{\circ} \mathrm{C}$ for 10 minutes, followed by 40 cycles at $95^{\circ} \mathrm{C}$ for $15 \mathrm{sec}$ and $60^{\circ} \mathrm{C}$ for 1 minute. Real-time PCR assays were carried out in duplicate for each sample and mean values were used for the calculation of the mRNA levels. Real-time quantitative RT-PCR for CD90 was performed using Taqman gene expression probes (Applied Biosystems). Expression of mRNA was assessed using standard curve and normalized to $\beta$-actin. Data were expressed as relative expression units. ${ }^{20-21}$

\section{Statistical Methods}

Data was analyzed using SPSS win statistical package version 17 (SPSS Inc., Chicago, IL). Numerical data were expressed as mean and standard deviation or median and range as appropriate. Qualitative data were expressed as frequency and percentage. Chi-square test (Fisher's exact test) was used to examine the relation between qualitative variables. For quantitative data, comparison between two groups was done using Mann-Whitney test. OS was estimated from date of diagnosis till death while DFS from first date showing objective response $(\mathrm{CR} / \mathrm{PR})$ till disease relapse or progression. Survival analysis was done using Kaplan-Meier method and comparison between two survival curves was done using log-rank test. Cox-regression method was used to test survival for numeric variables and $p$ value $<0.05$ was considered significant.

\section{Results}

Twenty six males and 17 females (1.5:1) were included in the current study with a median age of 24 months (range: 2 months to 11 years). In 31/43 tumor was located at the right lobe, $7 / 43$ at the left lobe, and 5/43 had a multifocal hepatic tumor. Stage I disease was present in $22(51.2 \%)$ of our study patients, 1 patient (2.3\%) had stage II, 16 (37.2\%) stage III, and 4 patients $(9.3 \%)$ with stage IV disease had lung metastasis at time of presentation. Histological variants included fetal subtype in 26 (60.5\%), embryonal in 4 (9.3\%), and mixed morphology in $13(30.2 \%)$ patients.

Twenty one patients had upfront tumor resection (20 stage I and 1 stage II) while all stage III and IV patients $(n=20)$ in addition to another 2 patients with stage I tumor received neoadjuvant chemotherapy. Eventually, surgical resection was feasible in 5 initially inoperable patients with stage III following 4 cycles of chemotherapy and after 2 cycles only in another 2 patients with stage I disease. Adjuvant postoperative chemotherapy was given to 10 patients for stage I other than purely fetal and low mitotic index histology and 1 with stage II disease.

By the end of their upfront treatment (either surgery or neoadjuvant chemotherapy), 20/43 patients (46.5\%) attained complete response (CR) after initial surgery, all of them had stage I disease whereas 23 patients $(53.5 \%)$ did not; 7 had partial response (PR) $[1$ had stage II with postoperative positive surgical margins, 2 were stage I and 4 were stage III who received neoadjuvant chemotherapy], 5 patients showed disease progression (PD) [4 of them were stage IV and a single patient had stage III], while 11 stage III patients had stationary disease (SD). The 4 years OS and DFS for all patients under study were $58.2 \%$ and $82.4 \%$ respectively.

\section{Markers expression}

CD44 protein and RNA were reported in 21 (48.8\%) and 20 (46.5\%) patients respectively; with $85.7 \%$ concordance. CD90 protein and RNA were reported in 14 (32.6\%) and 18 (41.7\%) 
cases respectively; with $77.8 \%$ concordance whereas, CD133 protein and RNA were reported in 21 (48.8\%) and 25 (58.1\%) patients respectively; with $96 \%$ concordance [Figure 1]. For statistical purposes, only cases showing both protein and RNA expression were scored positive. Increased expression of all studied markers (CD44, CD90, CD133) was significantly correlated with; higher disease stages III and IV versus stages I and II $(p<0.001)$; and with poor response to treatment $\mathrm{PR} / \mathrm{SD} / \mathrm{DP}$ in comparison to CR $(p<0.001)$.

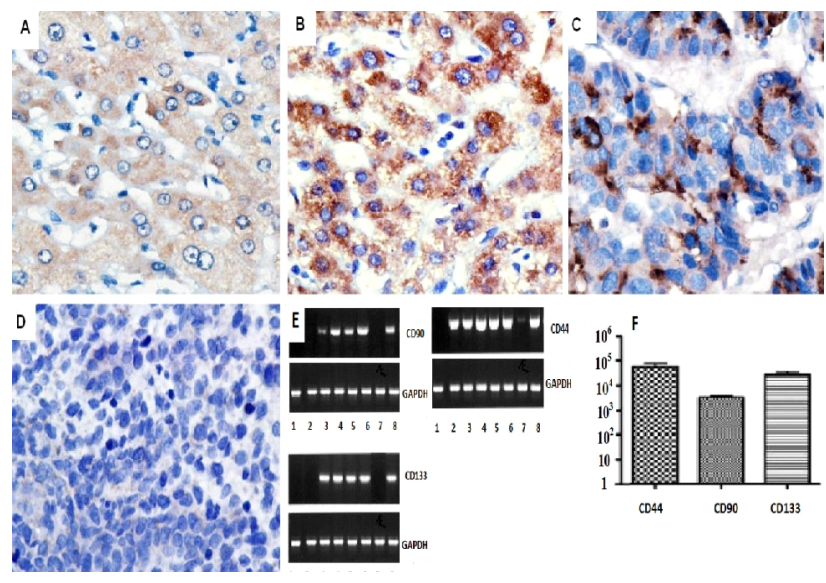

FIG.1 (A-D): Cases of hepatoplastoma showing positive membrano-cytoplasmic immunostaining for CD44 (A), CD90 (B), CD133 (C), and a negative control (D). (E\&F): RT/PCR analysis of CD44, CD90 and CD133 with GAPDH as a housekeeping gene in the hepatoblastoma cases.

\section{Survival correlations}

The OS was significantly correlated with disease stage $(p<$ 0.001). Patients underwent total surgical tumor resection (12 segmentectomy \&12 lobectomy) showed a significantly better OS compared to those who had incomplete or no resection ( $p$ $<0.001$; Table 1).

As regards CSC markers expression, cases with positive CD90, CD133, and CD44 expression (each or all) showed a significantly lower OS than negative cases $(p<0.001$; Figure 2$)$, whereas only CD133 and CD44 expression were significantly correlated with DFS ( $p=0.03$ and $p<0.001$, respectively) [Table 1 and Figure 3].

On multivariate analysis, only complete surgical resection and CD90 expression were independently predictive of OS (OR 7.9 and 11.8 respectively), whereas CD44 only found to be an independent prognostic factor for DFS (OR 15.8).
TABLE 1: Correlation of Clinicopathological Variables and CSCs Markers to Survival at 4 Years

\begin{tabular}{|c|c|c|c|c|c|c|}
\hline & \multicolumn{3}{|c|}{ Overall Survival } & \multicolumn{3}{|c|}{ Disease Free Survival } \\
\hline Variable & No. & $(\%)$ & $p$ & No. & $(\%)$ & $P$ \\
\hline Age & & & & & & \\
\hline$\leq 24$ months & 22 & 48.6 & & 13 & 81.8 & \\
\hline$>24$ months & 21 & 75.1 & 0.23 & 14 & 82.5 & 0.93 \\
\hline Gender & & & & & & \\
\hline Male & 26 & 61.3 & & 14 & 85.1 & \\
\hline Female & 17 & 49.1 & 0.49 & 13 & 78.7 & 0.73 \\
\hline Stage & & & & & & \\
\hline Stage I\&II & 23 & 81.9 & & 21 & 83.5 & \\
\hline Stage III\&IV & 20 & 30.0 & $<0.001$ & 6 & 75.0 & 0.58 \\
\hline Histology & & & & & & \\
\hline Fetal & 26 & 68.7 & & 16 & 85.7 & \\
\hline Other subtypes & 17 & 41.8 & 0.46 & 11 & 77.8 & 0.48 \\
\hline $\begin{array}{l}\text { Complete surgical } \\
\text { resection }\end{array}$ & & 25.2 & & & * & \\
\hline No & 19 & 83.8 & & 5 & 84.4 & \\
\hline Yes & 24 & & $<0.001$ & 22 & & 0.33 \\
\hline Nodes & & & & & & \\
\hline Negative & 35 & 56.1 & & 21 & 89.1 & \\
\hline Positive & 8 & 65.6 & 0.56 & 6 & ${ }^{*}$ & 0.04 \\
\hline Initial Response & & & & & & \\
\hline CR & 20 & 100 & & & & \\
\hline $\mathrm{PR}$ & 7 & 41.6 & & & $* *$ & $* *$ \\
\hline $\mathrm{SD} / \mathrm{PD}$ & 16 & 16.1 & $<0.001$ & & & \\
\hline CD 90 & & & & & & \\
\hline Negative & 29 & 79.3 & & 25 & 85.2 & $* * *$ \\
\hline Positive & 14 & 10.7 & $<0.001$ & 2 & 50.0 & \\
\hline CD 133 & & & & & & \\
\hline Negative & 22 & 95.4 & & 21 & 88.8 & \\
\hline Positive & 21 & 0 & $<0.001$ & 6 & 53.3 & 0.03 \\
\hline CD 44 & & & & & & \\
\hline Negative & 22 & 79.5 & & 20 & 94.1 & \\
\hline Positive & 21 & ${ }^{*}$ & $<0.001$ & 7 & $*$ & $<0.001$ \\
\hline
\end{tabular}

${ }^{*}$ No patients available at the predicted time interval.

${ }^{* *}$ No relation could be made as response is a base of entry for DFS

${ }^{* * *}$ Small number of patients in sub-groups. CSCs: cancer stem cells, CR: complete response, PR: partial response, SD: stable disease, PD: progressive disease, $\mathrm{CD}$ : cluster determinant

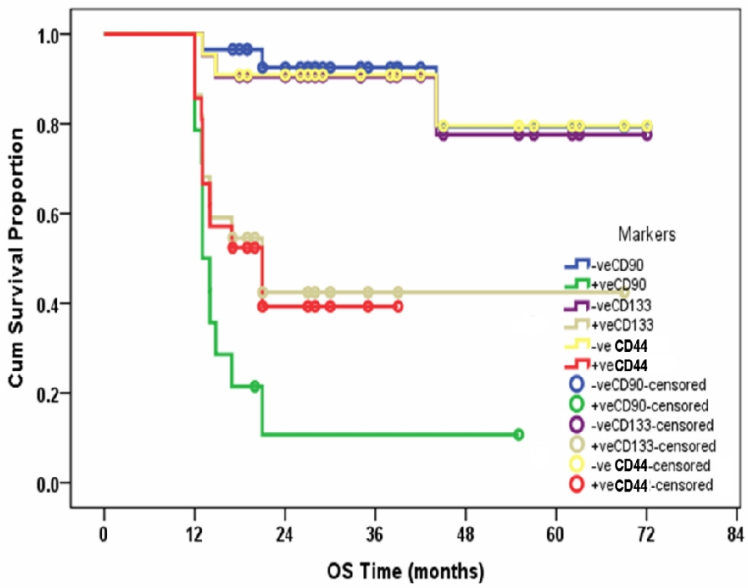

FIG. 2: Overall survival for $\mathrm{HB}$ patients in relation to CD44, CD90 \& CD133 $(p<0.001)$. 


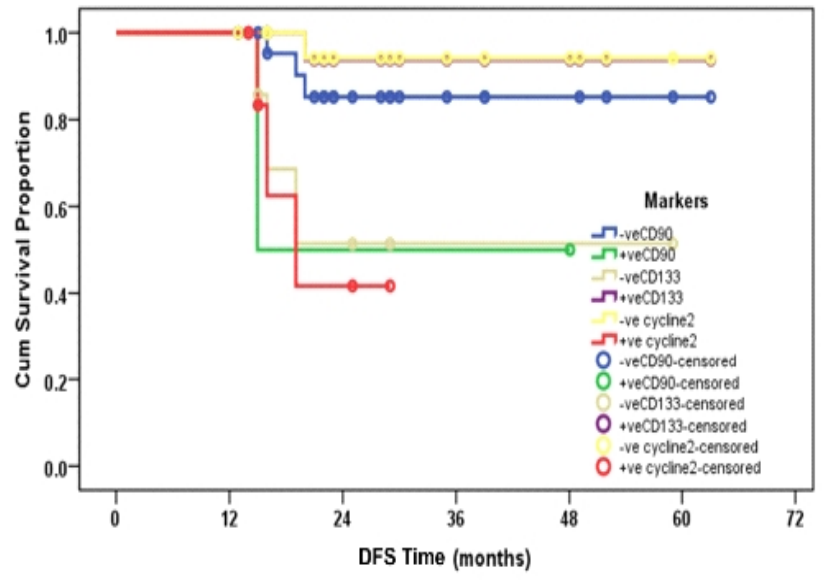

FIG. 3: Disease free survival for hepatoblastoma patients in relation to CD44, CD90 and CD133 expression $(p<0.001)$.

\section{Discussion}

Hepatoblastoma is a childhood liver cancer associated with several prognostic and predictive factors. To date, only few available case-control studies on small sample sizes discussing these factors including the premature birth, very low birth weight, histology subtype and disease stage. ${ }^{22-23}$

Matched to our results, males are affected more than females with HB despite possible ethnic variability in the United States. ${ }^{19}$ The prognostic impact of the pure fetal histological HB subtype was shown by other investigators to be associated with lower stages, more resectability, and better survival. ${ }^{22}$ In our study, patients with pure fetal histology had nominally higher OS rate $(68.7 \%)$ compared to those with other histological subtypes (41.8\%) but was not of statistical significance $(p=0.46)$. The lack of any significant difference among our patients could be attributed to the small number of patients within subgroups.

Although complete surgical resection is considered as the most important factor predicting patients who would achieve cure, yet chemotherapy response may also represent an important factor influencing survival in those patients. ${ }^{24}$

As recommended by the German group of Pediatric Oncology Hematology (GPOH) that primary resection in small liver tumors should be always attempted, investigators from Japan similarly favored the use of initial surgical resection whenever feasible followed by adjuvant chemotherapy to minimize overall chemotherapy exposure and toxicity. ${ }^{8,25}$ On the contrary, the Childhood Liver Tumors Strategy Group (SIOPEL) group has used the strategy of delayed surgery following initial chemotherapy in order to make the tumor smaller in size, less prone to bleed, and thus more likely to be completely resected. ${ }^{26}$

By the 1990s, the overall survival for HB had increased from $35 \%$ to $70 \%$ and has further increased to nearly $80 \%$ in the most recent trials. In some series overall survival was more than $90 \%$ for a localized disease. ${ }^{24}$ In two recent studies, a long-term survival in patients with advanced disease stages ranged from $30 \%$ to $60 \%$ using systemic chemotherapy. ${ }^{8}$ Patients with advanced stages (III\&IV) in our study had $30 \%$ OS in contrast to those with a more localized disease (stage I\&II) showing a significantly better OS (81.9\%); $(p<0.001)$. The better survival outcome in patients with early stage disease could be partly attributed to the fact that those patients were usually amenable to surgical resection of their tumors. In accordance, OS of our patients with total surgical resection was superior to those with incomplete or no resection $(83.8 \%$ vs. $25.2 \%$ respectively; $p<0.01$ ). The impact of tumor response on the OS was also shown in our study [Table 1].

The CSCs detected in several adult tumors, are characterized by multipotency, self-renewal, proliferation and maintenance of the neoplastic clone. Suggested role for CSCs in resistance to conventional chemo- and radiotherapy might be responsible for the aggressive behavior of these tumors. Therefore, therapies targeted to CSC markers and their mechanisms of resistance is an area of extensive research. ${ }^{27,28} \mathrm{In} \mathrm{HB}$, a side population (SP) was successfully isolated from a HB cell line which was able to form tumors in mice whereas tumors did not develop in the non-SP inoculated mice. ${ }^{13,29}$ However, a single study has been done on human clinical samples which included HCC and $4 \mathrm{HB}$ cases, of which one case only was positive for CSC markers. ${ }^{14}$ In the current study the CSC markers CD44, CD90 and CD133 proteins; were respectively expressed in $48.8 \%, 32.6 \%$ and $58.1 \%$ of the cases, compared to $46.5 \%, 32.6 \%$ and $41.7 \%$ for RNA. The expression of these markers was significantly associated with advanced disease stage, poor response to treatment and reduced survival indicating that these markers could be used as surrogate markers in HB patients and for targeted therapy which has been previously reported in other solid tumors but not in HB. ${ }^{30}$

\section{Conclusion}

Localized disease in hepatoblastoma is associated with a higher OS than the more advanced stages III and IV whereas, complete surgical resection of the primary tumor facilitated with preoperative chemotherapy in initially irresectable cases can improve the survival outcome in such patients. Some CSC markers (CD133, 44, and 90) were found to be predictive of both overall survival and response to treatment, however further studies are needed to confirm their prognostic role and their potential value as therapeutic targets. 


\section{Acknowledgement}

Most of gratitude goes to our patients whom we are learning from. Authors would also like to thank all physicians and nursing staff at the Pediatric Oncology Department, NCI, Cairo University who participated in care giving to our patients and to their families.

\section{Conflict of interest}

The authors declare that they have no conflicts of interest. The authors alone are responsible for the content and writing of the paper.

\section{References}

1. Meyers RL. Tumors of the liver in children. Surg Oncol 2007; 16:195-203.

2. Emre S, Umman V, Rodriguez-Davalos M. Current concepts in pediatric liver tumors. Pediatr Transplant 2012; 16:549-563.

3. Litten JB, Tomlinson GE. Liver tumors in children. Oncologist 2008; 13: 812-820.

4. Perilongo G, Shafford E, Plaschkes J; Liver Tumour Study Group of the International Society of Paediatric Oncology. SIOPEL trials using preoperative chemotherapy in hepatoblastoma. Lancet Oncol 2000; 1:94-100.

5. Stiller CA, Pritchard J, Steliarova-Foucher E. Liver cancer in European children: incidence and survival, 1978-1997. Report from the Automated Childhood Cancer Information System project. Eur $J$ Cancer 2006; 42:2115-2123.

6. McLaughlin CC, Baptiste MS, Schymura MJ, Nasca PC, Zdeb MS. Maternal and infant birth characteristics and hepatoblastoma. Am J Epidemiol 2006; 163: 818-828.

7. Boman F, Bossard C, Fabre M, Diab N, Bonnevalle M, Boccon-Gibod L. Mesenchymal hamartomas of the liver may be associated with increased serum alpha foetoprotein concentrations and mimic hepatoblastomas. Eur J Pediatr Surg 2004; 14:63-66.

8. Ortega JA, Douglass EC, Feusner JH, Reynolds M, Quinn JJ, Finegold MJ, et al. Randomized comparison of cisplatin/vincristine/fluorouracil and cisplatin/continuous infusion doxorubicin for treatment of pediatric hepatoblastoma: A report from the Children's Cancer Group and the Pediatric Oncology Group. JClin Orol2000; 18:2665-2675.

9. Zimmermann A. The emerging family of hepatoblastoma tumours: from ontogenesis to oncogenesis. Eur J Cancer 2005; 41:1503-1514.

10. Purcell R, Childs M, Maibach R, Miles C, Turner C, Zimmermann A, Sullivan M. HGF/c-Met related activation of $\beta$-catenin in hepatoblastoma. J Exp Clin Cancer Res 2011; 30:96.

11. Dalerba P, Cho RW, Clarke MF. Cancer stem cells: models and concepts. Annu Rev Med 2007; 58:267-284.

12. Park CY, Tseng D, Weissman IL. Cancer stem cell-directed therapies: recent data from the laboratory and clinic. Mol Ther 2009; 17:219-230.

13. Hayashi S, Fujita K, Matsumoto S, Akita M, Satomi A. Isolation and identification of cancer stem cells from a side population of a human hepatoblastoma cell line, HuH-6 clone-5. Pediatr Surg Int 2011; 27:9-16.

14. Lingala S, Cui YY, Chen X, Ruebner BH, Qian XF, Zern MA, Wu J. Immunohistochemical staining of cancer stem cell markers in hepatocellular carcinoma. Exp Mol Pathol 2010; 89:27-35.

15. Klonisch T, Wiechec E, Hombach-Klonisch S, Ande SR, Wesselborg S, Schulze-Osthoff K, Los M. Cancer stem cell markers in common cancerstherapeutic implications. Trends Mol Med 2008; 14:450-460.

16. McCarville MB, Roebuck DJ. Diagnosis and staging of hepatoblastoma: imaging aspects. Pediatr Blood Cancer 2012; 59:793-799.

17. King SJ, Babyn PS, Greenberg ML, Phillips MJ, Filler RM. Value of CT in determining the resectability of hepatoblastoma before and after chemotherapy. AJR Am J Roentgenol 1993; 160:793-8.

18. Therasse P, Arbuck SG, Eisenhauer EA, Wanders J, Kaplan RS, Rubinstein L, et al. New guidelines to evaluate the response to treatment in solid tumors (RECIST guidelines). J Natl Cancer Inst 2000; 92:205-216.

19. National Cancer Institute at the National Institutes of Health. Childhood Liver Cancer Treatment. Treatment of Hepatoblastoma. Available from http://www.cancer.gov/cancertopics/pdq/treatment /childliver/HealthProfessional/page5. (Accessed on March 29, 2013)

20. Khoo TK, Coenen MJ, Schiefer AR, Kumar S, Bahn RS. Evidence for Enhanced Thy-1 (CD90) Expression in Orbital Fibroblasts of Patients with Graves' Ophthalmopathy. Thyroid 2008; 18:1291-1296.

21. Olempska M, Eisenach PA, Ammerpohl O, Ungefroren $\mathrm{H}, \mathrm{F}$, Kalthoff $\mathrm{H}$. Detection of tumor stem cell markers in pancreatic carcinoma cell lines. Hepatobiliary Pancreat Dis Int 2007; 6:92-97.

22. Spector LG, Birch J. The Epidemiology of Hepatoblastoma. Pediatr Blood Cancer 2012; 59:776-779.

23. Howlader N, Noone AM, Krapcho M, Neyman N, Aminou R, Waldron W, et al. SEER cancer statistics review, (Vintage 2009 Populations), National Cancer Institute. Bethesda, MD 2011; 1975-2008.

24. Zsíros J, Maibach R, Shafford E, Brugieres L, Brock $\mathrm{P}$, Czauderna $\mathrm{P}$, et al. Successful treatment of 
childhood high-risk hepatoblatoma with dose-intensive multiagent chemotherapy and surgery: final results of the SIOPEL-3HR study. J Clin Oncol 2010; 28:2584-2590.

25. Tiao GM, Bobey N, Allen S, Nieves N, Alonso M, Bucuvalas J, et al. The current management of hepatoblastoma. A combination of chemotherapy, conventional resection, and liver transplantation. $J$ Pediatr 2005; 146:204-211.

26. Pritchard J, Brown J, Shafford E, Perilongo G, Brock P, Dicks-Mireaux C, et al. Cisplatin, doxorubicin, and delayed surgery for childhood hepatoblastoma: A successful approach-results of the first prospective study of the International Society of Pediatric Oncology. J Clin Oncol 2000; 18:3819-3828.
27. Garvalov BK, Acker T. Cancer stem cells: A new framework for the design of tumor therapies. $J \mathrm{Mol}$ Med 2011; 89:95-107.

28. Saini V, Shoemaker RH. Potential for therapeutic targeting of tumor stem cells. Cancer Sci 2010; 101:16-21.

29. Murase M, Kano M, Tsukahara T, Takahashi A, Torigoe T, Kawaguchi S, et al. Side population cells have the characteristics of cancer stem-like cells/cancer-initiating cells in bone sarcomas. $\mathrm{Br} J$ Cancer 2009; 101:1425-1432.

30. Frank NY, Schatton T, Frank MH. The therapeutic promise of the cancer stem cell concept. J Clin Invest $2010 ; 120: 41-50$. 